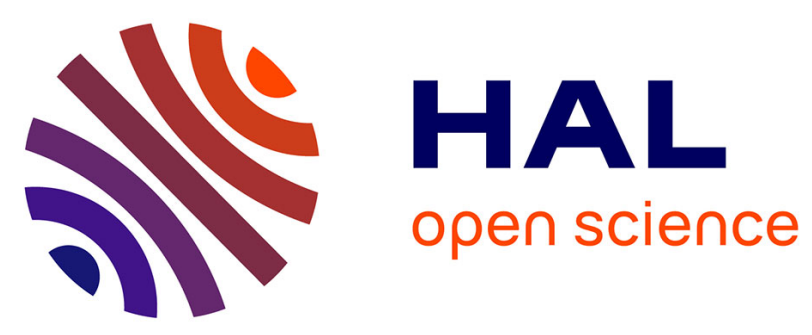

\title{
Dissecting Effects of Anti-cancer Drugs and Cancer-Associated Fibroblasts by On-Chip Reconstitution of Immunocompetent Tumor Microenvironments
}

\author{
Marie Nguyen, Adele de Ninno, Arianna Mencattini, Fanny Mermet-Meillon, \\ Giulia Fornabaio, Sophia Evans, Mélissande Cossutta, Yasmine Khira, Weijing \\ Han, Philemon Sirven, et al.
}

\section{- To cite this version:}

Marie Nguyen, Adele de Ninno, Arianna Mencattini, Fanny Mermet-Meillon, Giulia Fornabaio, et al.. Dissecting Effects of Anti-cancer Drugs and Cancer-Associated Fibroblasts by On-Chip Reconstitution of Immunocompetent Tumor Microenvironments. Cell Reports, 2018, 25 (13), pp.3884-3893.e3. 10.1016/j.celrep.2018.12.015 . hal-02343968

\section{HAL Id: hal-02343968 https://hal.science/hal-02343968}

Submitted on 3 Nov 2019

HAL is a multi-disciplinary open access archive for the deposit and dissemination of scientific research documents, whether they are published or not. The documents may come from teaching and research institutions in France or abroad, or from public or private research centers.
L'archive ouverte pluridisciplinaire HAL, est destinée au dépôt et à la diffusion de documents scientifiques de niveau recherche, publiés ou non, émanant des établissements d'enseignement et de recherche français ou étrangers, des laboratoires publics ou privés. 


\section{Cell Reports}

\section{Dissecting Effects of Anti-cancer Drugs and Cancer- Associated Fibroblasts by On-Chip Reconstitution of Immunocompetent Tumor Microenvironments}

\section{Graphical Abstract}

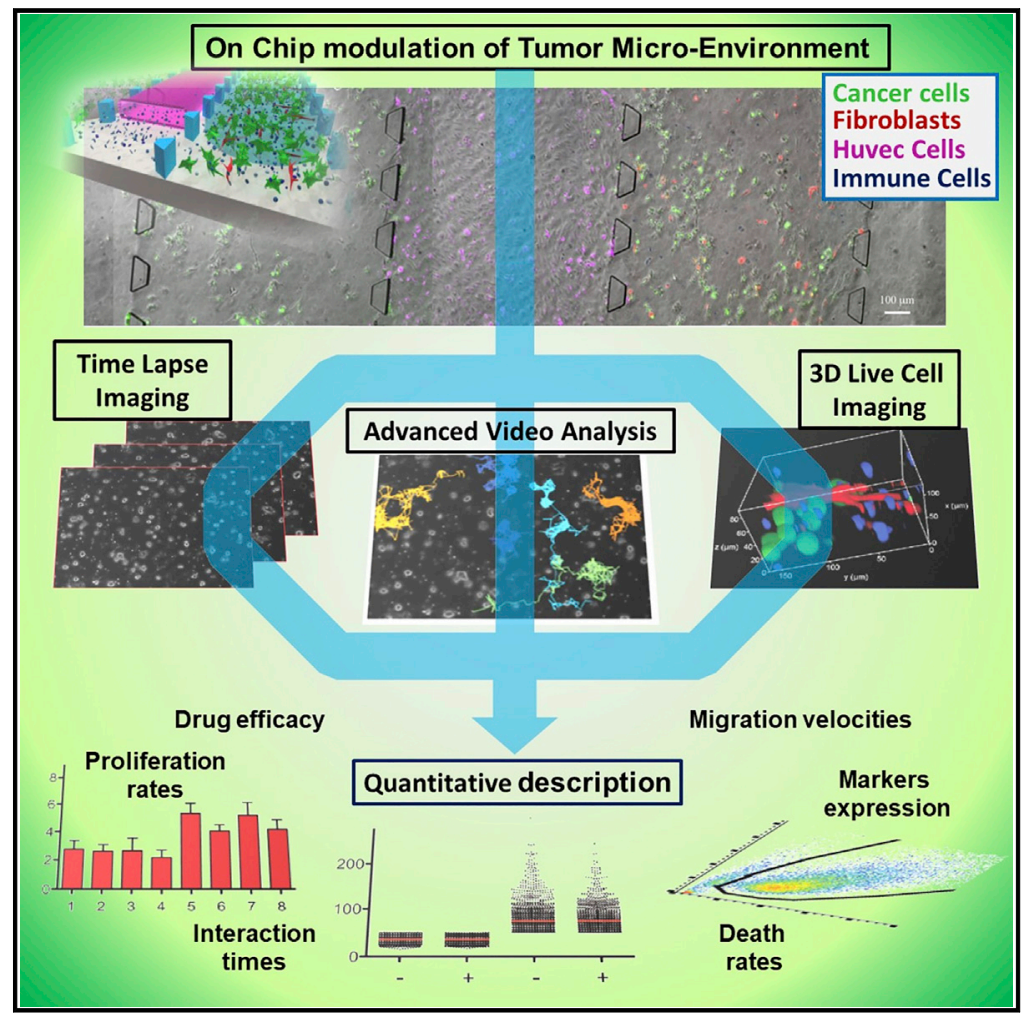

Highlights

- A complex HER2 ${ }^{+}$breast cancer ecosystem is reconstituted and quantitatively described

- The effects of the drug trastuzumab (Herceptin) are directly visualized ex vivo

- Trastuzumab promotes long cancer-immune interactions and an ADCC immune response

- Trastuzumab and CAFs have antagonist immunomodulation effects

\section{Authors}

Marie Nguyen, Adele De Ninno, Arianna Mencattini, ..., Eugenio Martinelli, Luca Businaro, Maria Carla Parrini

\section{Correspondence}

maria-carla.parrini@curie.fr

\section{In Brief}

Inspired by the emerging technology of tumor-on-chip, Nguyen et al. reconstituted ex vivo a human tumor microenvironment (HER2 ${ }^{+}$breast cancer), characterized the ecosystem-level responses to the drug trastuzumab (Herceptin), and dissected the roles of stromal components (immune cells and fibroblasts), demonstrating the power of immunocompetent tumors-on-chip for preclinical drug studies. 


\title{
Dissecting Effects of Anti-cancer Drugs and Cancer- Associated Fibroblasts by On-Chip Reconstitution of Immunocompetent Tumor Microenvironments
}

\author{
Marie Nguyen, ${ }^{1,2,11}$ Adele De Ninno, 3,9,11 Arianna Mencattini, ${ }^{4,11}$ Fanny Mermet-Meillon, ${ }^{1,2,11}$ Giulia Fornabaio, ${ }^{1,2}$ \\ Sophia S. Evans, ${ }^{1,2}$ Mélissande Cossutta, ${ }^{1,2}$ Yasmine Khira, ${ }^{1,2}$ Weijing Han, ${ }^{1,2}$ Philémon Sirven, ${ }^{1,5}$ Floriane Pelon, ${ }^{1,8}$ \\ Davide Di Giuseppe, ${ }^{4}$ Francesca Romana Bertani, ${ }^{3}$ Annamaria Gerardino, ${ }^{3}$ Ayako Yamada, $, 1,6,7$ Stéphanie Descroix, $1,6,7$ \\ Vassili Soumelis, ${ }^{1,5}$ Fatima Mechta-Grigoriou, ${ }^{1,8}$ Gérard Zalcman, ${ }^{1,2,10}$ Jacques Camonis, ${ }^{1,2}$ Eugenio Martinelli, ${ }^{4,12}$ \\ Luca Businaro, 3,12 and Maria Carla Parrini ${ }^{1,2,12,13, *}$ \\ ${ }^{1}$ Institut Curie, Centre de Recherche, Paris Sciences et Lettres Research University, 75005 Paris, France \\ ${ }^{2}$ ART Group, INSERM U830, 75005 Paris, France \\ ${ }^{3}$ Institute for Photonics and Nanotechnology, Italian National Research Council, 00156 Rome, Italy \\ ${ }^{4}$ Department of Electronic Engineering, University of Rome Tor Vergata, 00133 Rome, Italy \\ ${ }^{5}$ Immunity and Cancer, INSERM U932, INSERM Center of Clinical Investigations, CIC IGR Curie, 75005 Paris, France \\ 6 Laboratoire Physico Chimie Curie, CNRS UMR168, 75005 Paris, France \\ ${ }^{7}$ Institut Pierre-Gilles de Gennes, 75005 Paris, France \\ ${ }^{8}$ Stress and Cancer Team, labelized by Ligue Nationale Contre le Cancer, INSERM U830, 75005 Paris, France \\ 9 Department of Civil Engineering and Computer Science, University of Rome Tor Vergata, 00133 Rome, Italy \\ ${ }^{10}$ Centre d'Investigation Clinique (CIC) 1425, Hôpital Bichat-Claude Bernard, Université Paris-Diderot, Paris, France \\ ${ }^{11}$ These authors contributed equally \\ ${ }^{12}$ Senior authors \\ ${ }^{13}$ Lead Contact \\ *Correspondence: maria-carla.parrini@curie.fr \\ https://doi.org/10.1016/j.celrep.2018.12.015
}

\section{SUMMARY}

A major challenge in cancer research is the complexity of the tumor microenvironment, which includes the host immunological setting. Inspired by the emerging technology of organ-on-chip, we achieved 3D co-cultures in microfluidic devices (integrating four cell populations: cancer, immune, endothelial, and fibroblasts) to reconstitute ex vivo a human tumor ecosystem (HER2 ${ }^{+}$breast cancer). We visualized and quantified the complex dynamics of this tumor-onchip, in the absence or in the presence of the drug trastuzumab (Herceptin), a targeted antibody therapy directed against the HER2 receptor. We uncovered the capacity of the drug trastuzumab to specifically promote long cancer-immune interactions (>50 $\mathrm{min})$, recapitulating an anti-tumoral ADCC (antibody-dependent cell-mediated cytotoxicity) immune response. Cancer-associated fibroblasts (CAFs) antagonized the effects of trastuzumab. These observations constitute a proof of concept that tumors-on-chip are powerful platforms to study ex vivo immunocompetent tumor microenvironments, to characterize ecosystem-level drug responses, and to dissect the roles of stromal components.

\section{INTRODUCTION}

Tumor microenvironments are ecosystems composed of a variety of cell types (e.g., cancer cells, fibroblasts, immune cells, endothelial cells) positioned in complicated physicochemical contexts. It is now well established that understanding the complexity of the tumor ecosystems and their organizational principles is crucial, as they play a pivotal role in cancer progression, metastasis, and response to therapies (Klemm and Joyce, 2015). This complexity is commonly studied using animal models, usually immunodeficient mice to which human tumors are xenografted, with 2 major limitations: poor control of all variable factors and the impossibility of addressing questions about the role of the immune system. In addition, animal experimentation is long, expensive, and ethically questionable, and the transposition of results to humans is still debatable.

Recent advances in microtechnology inspired new strategies to reproduce ex vivo the complexity of biological systems. For example, functional units of some organs (e.g., lungs) and specific biological processes (e.g., angiogenesis) were reconstituted by placing human cells in microfluidic devices, giving rise to the field of organs-on-chip (Huh et al., 2013). These approaches mimic organ topography, functionality, and complexity much better than conventional 2-dimensional (2D) or even 3D culture systems (van Duinen et al., 2015). In the cancer field, pioneering works reported 3D microarchitectures on-chip that mimic certain features of the tumor microenvironments, building the basis for the technology of tumors-onchip (Bersini et al., 2014; Hassell et al., 2017; Sung and Beebe, 2014; Zervantonakis et al., 2012). In particular, our recent papers (Businaro et al., 2013; Parlato et al., 2017; Vacchelli et al., 2015) demonstrated how powerful these devices are in monitoring cancer-immune interactions. In these previous studies, the allogeneic nature of the immune cells did not apparently lead to major interferences, presumably because the coculture times were too short for a mixed lymphocyte reaction 
A

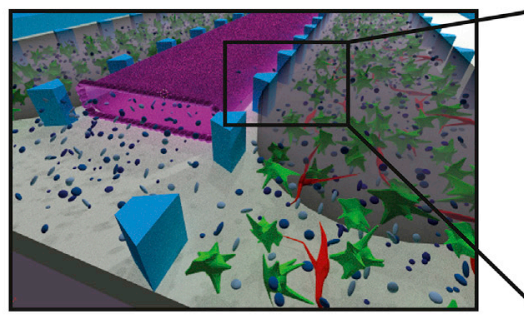

B

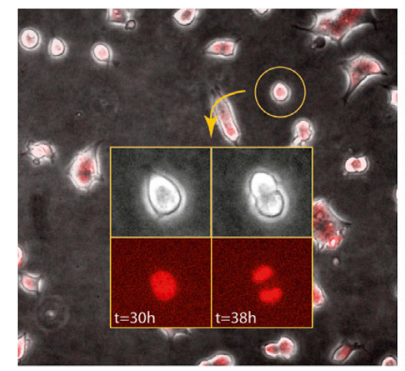

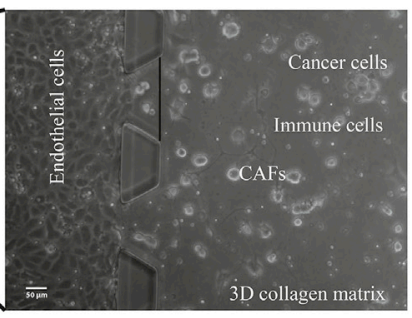

Counting Apoptosis

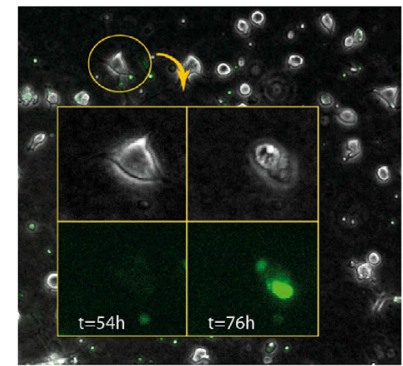

Figure 1. Reconstitution and Quantitative Description of an HER2 ${ }^{+}$Tumor Ecosystem with Four Cell Populations

(A) The tumor-on-chip approach. At left, a scheme of the chip, with a central vascular compartment made of a monolayer of endothelial cells (pink), 2 lateral chambers with 3D collagen I hydrogels (gray) in which cancer cells (green) and CAFs (red) are embedded, together with immune cells (blue). At right, a real image from a representative video is shown. Scale bar, $50 \mu \mathrm{m}$.

(B) Counting mitosis and apoptotic death of cancer cells. Representative events are shown. To monitor mitosis, nuclei were live stained using a far-red nuclear dye (SiR-DNA). To monitor death, cells were live stained using a green probe, which detects caspase 3 and 7 activity (CellEvent caspase-3/7).

(C) CellHunter method to track cancer-immune cell interactions. The method implies 3 steps: video acquisition, cell localization, and cell tracking.

dent cell-mediated cytotoxicity [ADCC]) (Arnould et al., 2006; Clynes et al., 2000; Kute et al., 2012; Varchetta et al., 2007).

In this work, we reconstituted the HER2 $^{+}$breast tumor microenvironment in 3D hydrogels on-chip containing a central endothelium compartment. We visualized and quantified the ecosystem dynamics (proliferation, apoptosis, motility, and cancer-immune cell-cell interactions) over 4 days, according to a defined stromal composition: with or without immune cells or with or without cancer-associated fibroblasts (CAFs).

We demonstrated that the presence of trastuzumab, in addition to inhibiting cancer cell proliferation and stimulating cancer cell death as expected, increased cancer-immune interactions and induced

(MLR) (Nguyen et al., 2003). Despite this huge potential, so far the use of tumors-on-chip has been restrained to a few specialized laboratories and has not reached the broad community of cancer research.

To validate the concept that tumors-on-chip could be exploited to study ex vivo responses to treatments that depend on the tumor ecosystem and to address oncoimmunology questions by monitoring complex multi-cell interactions involving immune cells, we chose the model of HER2 ${ }^{+}$breast cancer subtype (with HER2-receptor gene amplification) because of the availability of a targeted monoclonal antibody against the HER2 receptor, trastuzumab (brand name Herceptin). Trastuzumab has been a reference drug for patients with HER2 ${ }^{+}$breast cancers for more than a decade, with convincing overall survival benefits (Marty et al., 2005). It has been proposed that trastuzumab acts via not only a direct effect on cancer cells (inhibition of proliferation and induction of programmed cell death) but also an indirect effect mediated by the innate immune system (antibody-depen- an anti-tumoral ADCC immune response. In addition, we uncovered an inhibitory role for CAFs regarding cancer-immune interactions, supporting the emerging concept of an immune-suppressive role for CAFs in the tumor microenvironment (Costa et al., 2018; Feig et al., 2013; Givel et al., 2018; Kraman et al., 2010; Liao et al., 2009; Yang et al., 2016; Zhang and Ertl, 2016) and suggesting that CAFs may contribute to resistance to trastuzumab.

\section{RESULTS}

Reconstitution of an HER2+ Tumor Ecosystem On-Chip, Including CAFs and Immune Cells

We designed a microfluidic device for cell co-cultures (called a chip for short), based on published works (Chen et al., 2017; Lucarini et al., 2017; Parlato et al., 2017; Zervantonakis et al., 2012) (Figure 1A). The chips were microfabricated by soft lithography using PDMS (polydimethylsiloxane), a silicone rubber that is 

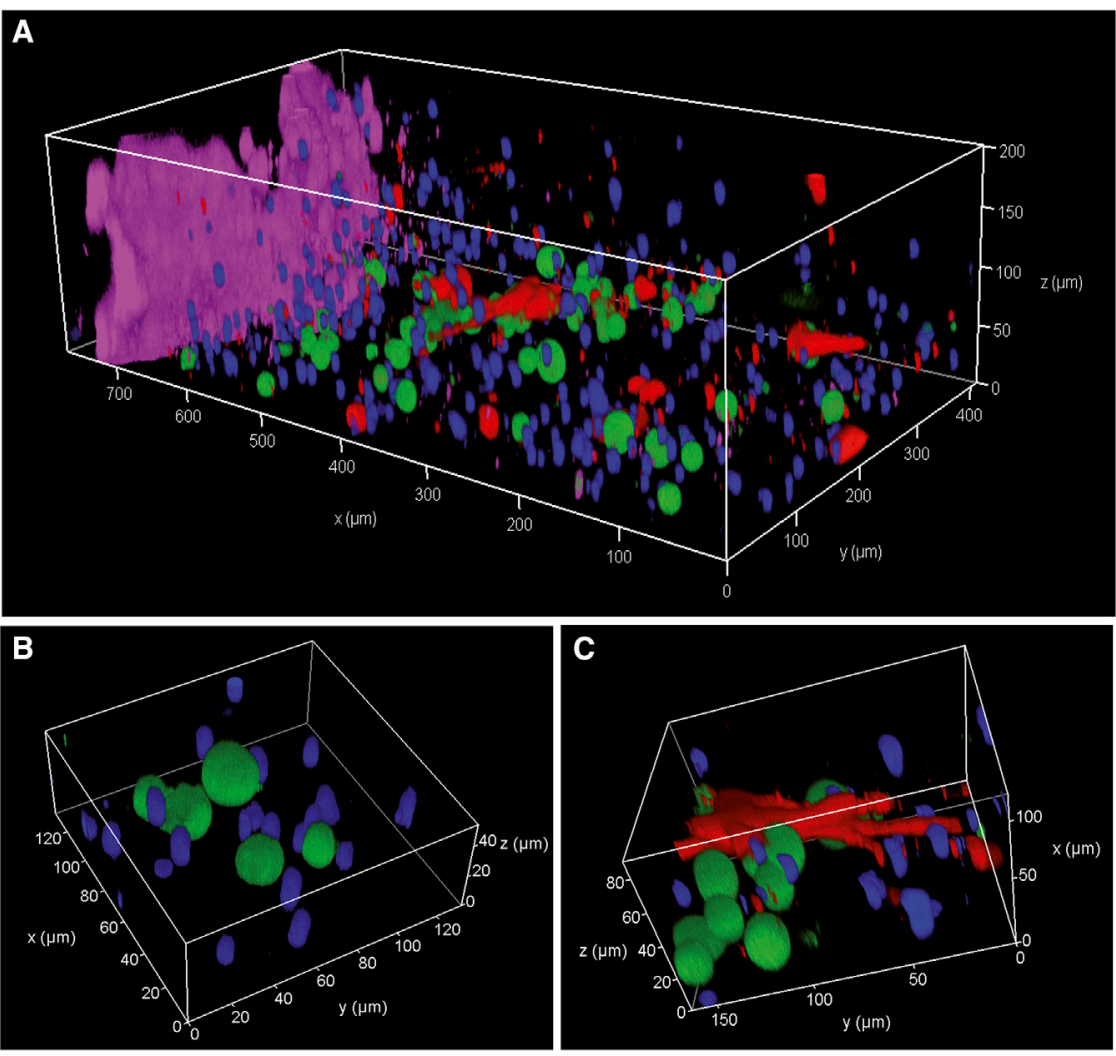

Figure 2. Confocal Imaging of the Reconstituted Tumor Ecosystem

(A) The various cell types were differentially live stained with CellTrace dyes before seeding inside the tumor-on-chip. The confocal image reconstruction shows the 3D positioning of BT474 cancer cells (green), Hs578T CAF cells (red), PBMC immune cells (blue), and HUVECs (violet).

(B) This magnified 3D view shows physical cell-cell interactions between BT474 cancer cells (green) and immune cells (blue).

(C) This magnified 3D view shows a CAF cell (red), which is contacting several cancer cells (green) and immune cells (blue)

the organ-on-chip terminology even for systems without perfusion, we adopted the tumor-on-chip terminology for our approach.

These tumors-on-chip were visualized by high-content video-microscopy (multipositioning, multi-colors, 2- to 120-min time intervals) for 4-5 days (Video S1). To discriminate cancer cells from CAFs, the fibroblasts were pre-stained in red with a live dye (CellTrace Yellow reagent) (Video S2). In addition, to quantitatively describe the tumor ecosystem, we implemented appropriate methods to measure on-chip proliferation, apoptotic death, and cell-cell inter-

biocompatible, gas permeable, and transparent. The chip design consisted of 5 parallel microchambers (500- to $1000-\mu \mathrm{m}$ wide, 150- to $200-\mu \mathrm{m}$ high), separated by regularly spaced micropillars that allow the confinement of hydrogels by means of a balance between surface tension and capillary forces. The various cell types were positioned inside the chips, mimicking their original in vivo architecture in tumors.

Cancer cells (the HER2 ${ }^{+}$breast cancer BT474 cell line), cancer-associated fibroblasts (the breast CAF cell line Hs578T), and immune cells (PBMCs [peripheral blood mononuclear cells] from healthy donors) were embedded into 3D biomimetic hydrogels (made of collagen type I at $2.3 \mathrm{mg} / \mathrm{mL}$, the major component of the extracellular matrix [ECM]), inside the 2 inner lateral chambers. To compare conditions with and without CAFs within the same chip, the left gel chamber was without CAFs, while the right gel chamber was with CAFs. The 2 outer lateral chambers were used as medium reservoirs. This work was focused on the effects of CAFs and immune cells; however, endothelial cells were always included since in vivo they contribute to shaping the biochemical environment by secreting a variety of cytokines and other soluble factors (Buchanan et al., 2012; Lee et al., 2015). Endothelial cells (primary human umbilical vein endothelial cells [HUVECs]) were grown as 2D monolayers in the central chamber, as previously reported (Zervantonakis et al., 2012). For simplicity, the majority of co-cultures and observations were performed without adding perfusion in this vessel compartment. Considering that $3 \mathrm{D}$ cell co-cultures were achieved in microfluidics devices and that the literature extensively uses actions (Figures 1B and 1C). To monitor proliferation, nuclei were live stained using a far-red nuclear dye (SiR-DNA), which allows for the identification and counting of mitotic events (Video S3). To monitor cell death, apoptotic cells were identified using a green probe detecting caspase 3 and 7 activity (CellEvent caspase-3/7) (Video S4). Cells undergoing apoptosis, in addition to emitting green fluorescence, also showed a change in morphology. The overall observation of hundreds of videos revealed extremely rich and complex cell-cell interactions involving immune cells (Video S5). Using the ad hoc-designed CellHunter automated method (Biselli et al., 2017; Parlato et al., 2017), we tracked the cell dynamics within the co-cultures and measured the interaction times with each immune cell for each cancer cell (Figure 1C), thus providing a high-content description of the cancer-immune cell interactions inside the reconstituted tumor ecosystem. The relative positioning and morphologies of the different cell populations were investigated by live snapshot confocal microscopy; cancer cells, immune cells, and fibroblasts are distributed along the $z$ dimension of the collagen gel, and several cell-cell contacts occur in this true 3D ecosystem. The endothelial cells create vertical barriers, although not continuous ones, at the interfaces between the endothelium channel and the collagen gels (Figure 2).

We modulated this experimental setting by adding or removing specific cellular components (CAFs and immune cells) and the drug trastuzumab, the invariable components being cancer and endothelial cells; in this way, we analyzed 8 different co-culture conditions, which are summarized in Figure 3. 
A

\begin{tabular}{|c|c|c|c|c|c|c|c|c|}
\hline & Condition 1 & Condition 2 & Condition 3 & Condition 4 & Condition 5 & Condition 6 & Condition 7 & Condition 8 \\
\hline Cancer cell & + & + & + & + & + & + & + & + \\
\hline CAF & - & + & - & + & - & + & - & + \\
\hline PBMCs & - & - & + & + & - & - & + & + \\
\hline HUVEC & + & + & + & + & + & + & + & + \\
\hline $\begin{array}{c}\text { Trastuzumab } \\
\mathbf{1 0} \mu \mathrm{g} / \mathrm{mL}\end{array}$ & - & - & - & - & + & + & + & + \\
\hline
\end{tabular}

B
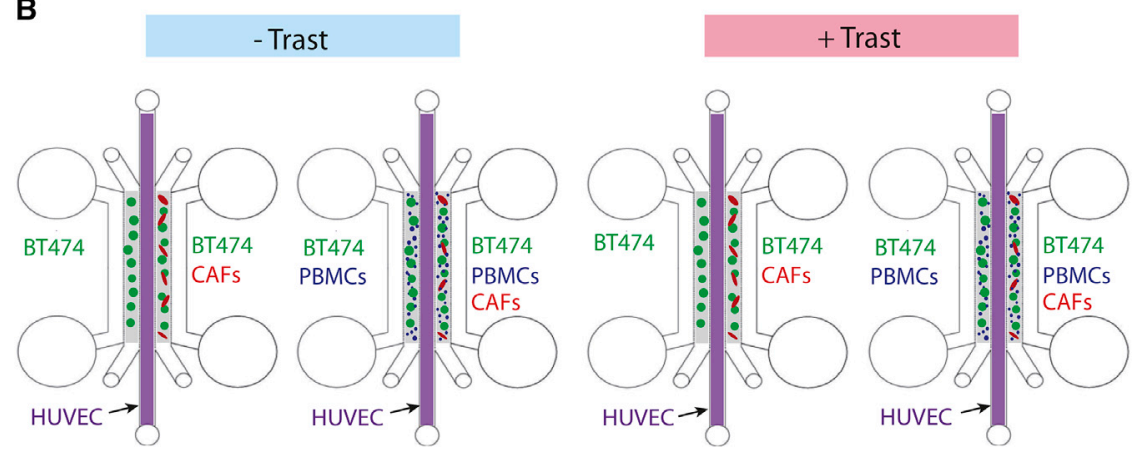

Figure 3. Experimental Design

(A) Summary of co-culture conditions.

(B) Experimental setting. The chips have a central endothelium compartment, where HUVECs are seeded (violet), and 2 gel compartments (gray), where BT474 cancer cells (green) are embedded, with or without Hs578T CAF cells (red) and PBMC immune cells (blue), depending on the conditions. To compare conditions with and without CAFs, the left gel chamber was without CAFs, and the right gel chamber was with CAFs. To compare conditions with and without PBMC immune cells, parallel chips were used in the same experiment. To compare conditions with and without trastuzumab, the drug was added at the beginning of the cocultures in the medium of all 3 chambers without gels: the endothelium central chamber and the 2 outer lateral chambers. Video-microscope acquisitions were initiated $3-24 \mathrm{hr}$ after the start of co-culture.

and the absence of trastuzumab $(\sim 20 \%$ increase) (Figures 4B, S1, and S2). The addition of trastuzumab led to a modest

Pro-invasive Effects of CAFs in the HER2 ${ }^{+}$Tumor Ecosystem

CAFs promote the motility and invasion of cancer cells by a multiplicity of possible mechanisms (Attieh et al., 2017; De Wever et al., 2004; Gaggioli et al., 2007; Glentis et al., 2017; Goetz et al., 2011; Labernadie et al., 2017). The heterogeneity of CAFs was very recently characterized in breast and ovarian cancers, identifying at least 4 different CAF subpopulations, referred to as CAF-S1-CAF-S4, with distinct properties and levels of activation (Costa et al., 2018; Givel et al., 2018). By using the same markers as in the aforementioned studies (epithelial cell adhesion molecule [EPCAM], fibroblast activation protein [FAP], CD29, platelet-derived growth factor subunit B [PDGFB], alpha smooth muscle actin [aSMA]), we characterized by fluorescence-activated cell sorting [FACS] the molecular identity of our CAF cell model, the Hs578T cell line, and found that it corresponds to the CAF-S1 subtype (Figure 4A). Pathway enrichment analysis had previously pointed out that genes upregulated in the CAF-S1 subset were involved in cell adhesion, ECM organization, and immune response; in particular, the CAF-S1 subset was shown to specifically promote immunosuppression through a multistep mechanism (Costa et al., 2018; Givel et al., 2018).

We questioned whether the presence of Hs578T CAF-S1 cells modulated cancer cell motility within the collagen type I matrix in the tumor-on-chip. We measured the movements of the BT474 cancer cells inside the tumors-on-chip by tracking these cells with CellHunter, in the presence or the absence of CAFs (conditions 3, 4, 7, and 8). Figure 4B shows representative results from 1 experiment ( $n$ tracks per condition $>600$ ), which were reproduced in 3 independent experiments (Figure S1). Similar results were obtained with manual tracking ( $n$ cells per condition $=45-65$ ) (Figure S2).

The presence of CAFs stimulated the velocities of BT474 cells, in a robust and highly reproducible manner, both in the presence but statistically significant increase in the velocities of BT474 cells, both with and without CAFs (Figures 4B, S1, and S2). In our ecosystem, the interactions between CAFs and BT474 cancer cells were rare and transitory (Video S2), which argues against direct pulling mechanisms by the highly motile CAFs on sluggish cancer cells and suggests the existence of longrange mechanisms of communication, such as via secreted molecules or ECM remodeling.

\section{Direct Visualization of Trastuzumab Effects on the HER2 ${ }^{+}$Tumor Ecosystem}

Trastuzumab is expected to specifically affect the growth of cells overexpressing the HER2 receptor. In standard 2D cultures, we verified that trastuzumab did inhibit the growth of HER2 ${ }^{+}$BT474 cells, but not that of the luminal MCF7 breast cancer cell line (Figure S3). The drug was used at a $10-\mu \mathrm{g} / \mathrm{mL}$ concentration, which likely corresponds to a clinically relevant range since it is on the same order of magnitude as the median concentration measured in the plasma of trastuzumab-treated patients $(60 \mu \mathrm{g} / \mathrm{mL})$ (Jamieson et al., 2009). This concentration was saturating since the half-maximal inhibitory concentration $\left(\mathrm{IC}_{50}\right)$ for the antiproliferative effects of trastuzumab on BT474 cells is $1.18 \mu \mathrm{g} / \mathrm{mL}$ (Shim et al., 2012). We showed that as a negative control, as expected, $10 \mu \mathrm{g} / \mathrm{mL}$ trastuzumab did not have any impact on the 2D growth of CAFs or HUVECs (Figure S3).

When $10 \mu \mathrm{g} / \mathrm{mL}$ trastuzumab was added to the HER2 ${ }^{+}$tumoron-chip medium (in central and 2 outer chambers), we observed a cytostatic effect, evaluated as a reduction $(\sim 0.4-$ fold $)$ in the percentage of BT474 mitoses per day (Figure 5A). Without the drug, the BT474 cancer cell population doubling time on-chip was approximately 10 -fold slower than in 2D (10-20 days versus 1-2 days). We speculate that the reduced rate may be due to the fact that the cells are embedded in the gel and that they must divide in a "real 3D" context. Of note, while very 
A

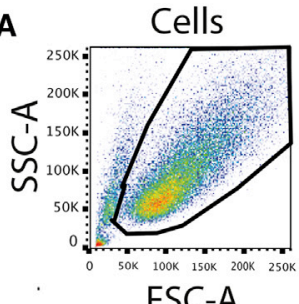

FSC-A

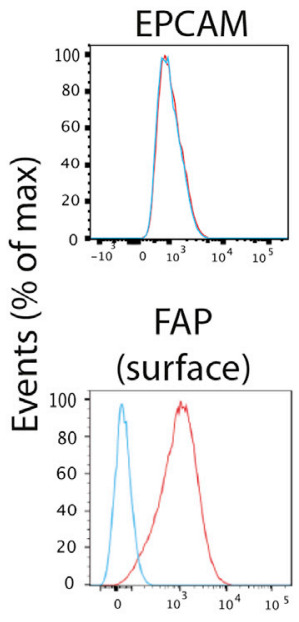

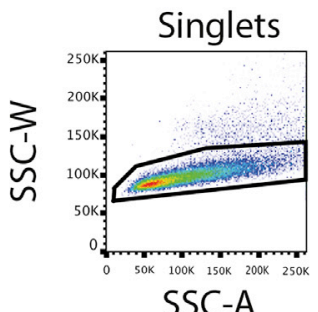

SSC-A

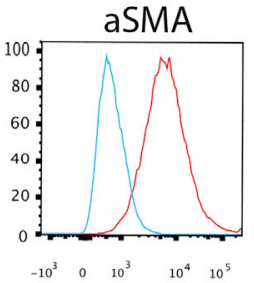

FAP

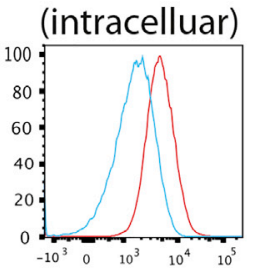

Fluorescence intensity

B
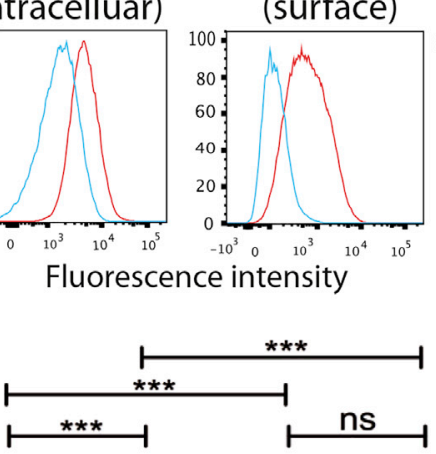
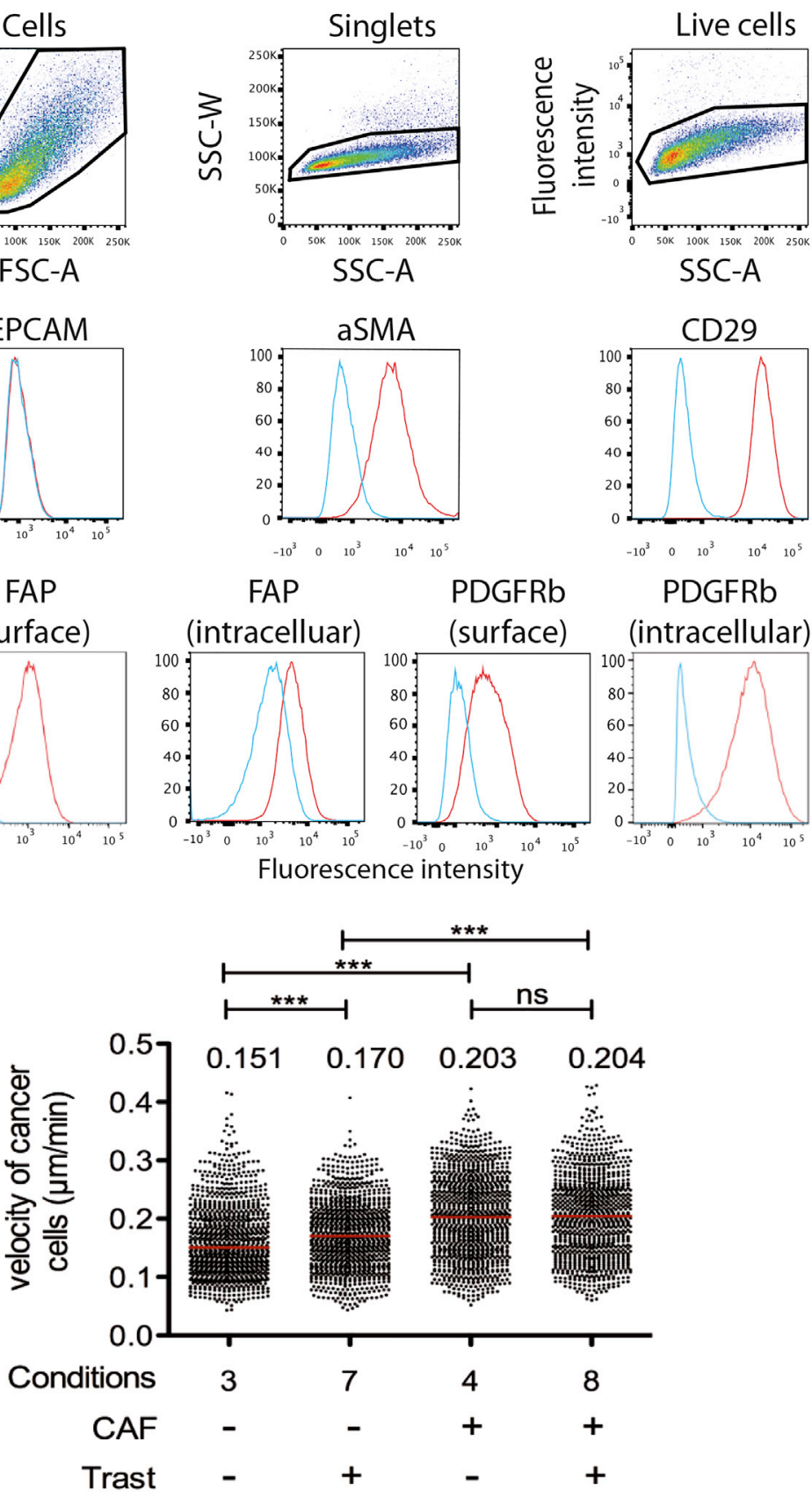

PDGFRb

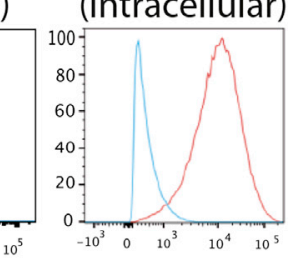

Figure 4. Characterization of the Hs578T CAF Cell Line

(A) CAF-S1 identity of the Hs578T cell line. Using flow cytometry analysis, Hs578T cells were first gated based on forward (FSC-A) and side (SSC-A) scatters (measuring cell size and granulosity, respectively) to exclude debris. Single cells were next selected based on SSC-A versus SSC-W parameters. Dead cells were excluded based on their positive staining for live/dead (for intracellular staining) or DAPI (for surface staining). Cells were next examined using 1 epithelial marker (EPCAM) and 4 CAF markers (aSMA, CD29, FAP, and PDGFB, the last 2 both intracellular and at surface, as indicated). Blue lines are isotype controls, while red lines are staining with specific antibodies. Hs578T cells are clearly $\mathrm{EPCAM}^{-}$, $\mathrm{aSMA}^{+}, \mathrm{CD}^{2} 9^{+}, \mathrm{FAP}^{+}$, and PDGFB $^{+}$.

(B) Pro-invasive effects of CAFs in the HER2 ${ }^{+}$tumor ecosystem. Velocity of BT474 cancer cells was measured for the indicated conditions. Each point represents the track of a cancer cell. The median values are shown by red bars and explicitly written above the points. Results from 1 representative experiment. Number of tracks $n>600$ per condition. ${ }^{*} p<0.05,{ }^{* *} p<0.001,{ }^{* \star *} p<0.0001$; ns, not significant, using Kolmogorov-Smirnov tests.

In the majority of the experiments presented in this work, the overall cell density was kept moderate $\left(1 \times 10^{6}\right.$ cells $/ \mathrm{mL}$ for cancer cells, $0.25 \times 10^{6}$ cells $/ \mathrm{mL}$ for CAFs, $5 \times 10^{6}$ cells $/ \mathrm{mL}$ for PBMCs) to maintain compatibility with cell dynamics quantifications. Moreover, the proportion of the various cell types (roughly $25 \mathrm{CAFs}$ and 500 immune cells for 100 cancer cells) reflected the in vivo situation, as assessed by FACS analysis on dissociated breast tumor samples. Our FACS measurements indicated a median 2.3:1 ratio for immune cells versus cancer cells and a median 1:5.8 ratio for CAFs versus cancer cells (Table S1).

In these conditions, with a local immune cell-to-cancer cell ratio in the range of $2: 1-5: 1$, the presence or absence of immune cells or of CAFs did not have a noticeable impact on mitosis, apoptosis, and tumor growth rates (Figures 5A-5C). This was

heterogeneous, the estimated breast tumor doubling times in vivo in patients are on the order of tens or hundreds of days (Heuser et al., 1979; Yoo et al., 2015).

In addition, trastuzumab had a cytotoxic effect, evaluated as an increase ( 2.5-fold) in the percentage of apoptosis of BT474 cells per day (Figure 5B). This mild cytotoxic effect of trastuzumab (up to $5 \%$ apoptosis per day) is in agreement with previous data obtained by FACS on 2D BT474 cultures (Diermeier-Daucher et al., 2011; Nahta et al., 2004). As a consequence, the tumor growth, calculated by subtracting the apoptosis rate from the mitosis rate, was impaired by the presence of trastuzumab (Figure $5 \mathrm{C}$ ). somewhat surprising since, in the presence of trastuzumab, the addition of immune cells (PBMCs) was expected to increase the apoptotic death of BT474 cancer cells, which is consistent with $A D C C$. We reasoned that $A D C C$ efficacy may require high immune cell density. When we increased the immune cell-tocancer cell ratio to $25: 1$, we did not observe any effect on the apoptosis rate of cancer cells as compared to the conditions without or with low PBMC density in the absence of trastuzumab, but we did observe a massive apoptotic death of BT474 cancer cells in the presence of trastuzumab (Figure 5D; Video S6). This demonstrates that we could recapitulate and directly 


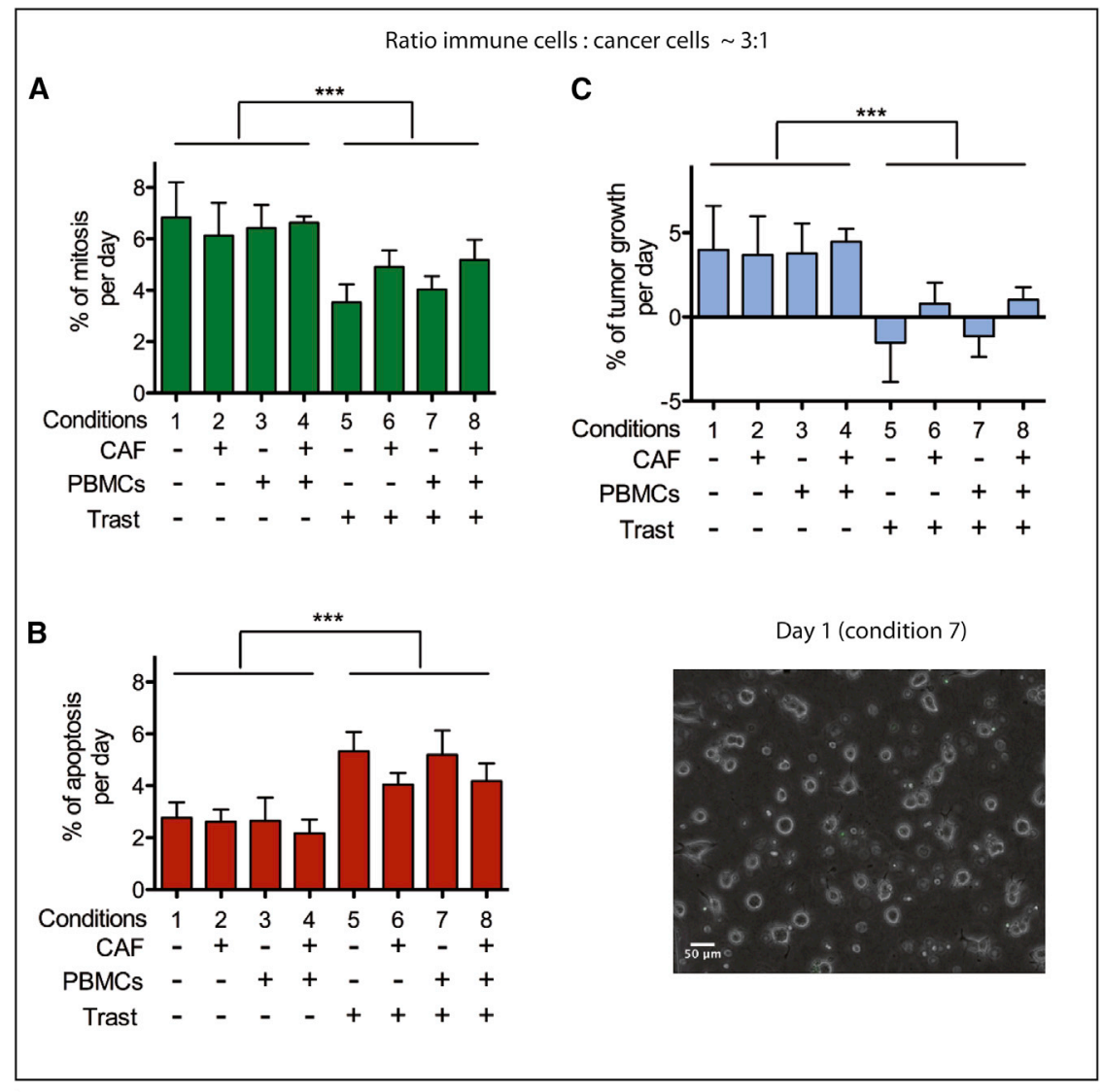

D Ratio immune cells : cancer cells $\sim 25: 1$

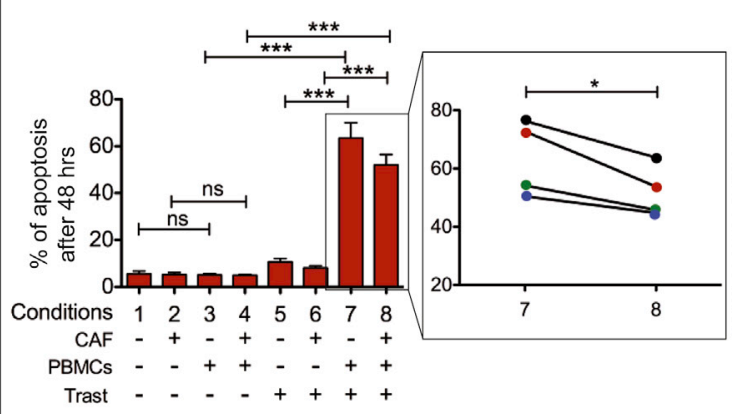

Day 1 (condition 7)

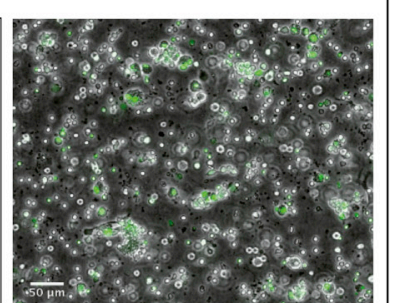

CACS - -++--+

Trast ---+++

Figure 5. Trastuzumab Effects on HER2 ${ }^{+}$ Tumor Ecosystem, Depending on Stromal Composition (Immune Cells and CAFs)

(A) Percentage of BT474 cells undergoing mitosis per day of observation.

(B) Percentage of BT474 cells undergoing apoptotic death per day of observation. Right: a representative image at day 1 of co-culture shows very few dying cells (green) for condition 7 .

(C) Calculated percentage of tumor growth per day of observation.

(D) Percentage of BT474 cells that died for apoptosis after $48 \mathrm{hr}$ of co-culture when the immune cell-to-cancer cell ratio was increased to $\sim 25: 1$. The magnified image shows the paired quantifications for each experiment (same PBMC donor), without and with CAFs. Right: a representative image at day 1 of co-culture shows many dying cells (green) for condition 7 .

Mitotic and apoptotic events were counted on the video images from 4 to 5 independent experiments. For clarity, the same apoptosis results for conditions 1,2, 5, and 6 were reported twice in (B) and (D). Number of cells $(n)=200-500$ per condition per experiment. The bars show means \pm SEMs. ${ }^{*} p<0.05,{ }^{* *} p<0.001,{ }^{* \star *} p<$ 0.0001 ; ns, not significant, using unpaired Student $t \mathrm{t}$ test, except for the magnification in (D), for which a paired $t$ test was used. Scale bars, $50 \mu \mathrm{m}$.

of the interaction times with the surrounding immune cells during the video observations (typically 3 days), using our CellHunter method (Figure 1C). Interaction is defined as a situation in which the distance between the center of a cancer cell and the center of an immune cell is less than twice the sum of the radii of the 2 cells.

The overall analysis of interactions, in 3 independent experiments, with 3 different PMBC donors, showed clear tendencies; the presence of trastuzumab appears to increase mean and maximum interaction times, while CAFs appear to decrease mean and maximum interaction times (Figure S4).

To better untangle these antagonist effects, we studied the time course impact

visualize on-chip the trastuzumab-induced ADCC (i.e., a drug response that depends on the immune system). Moreover, these results suggest that in vivo, the local density of infiltrating immune cells may modulate the ADCC response to trastuzumab. The presence of CAFs slightly inhibited the trastuzumab-induced ADCC, suggesting a potential immunomodulation effect mediated by CAFs (Figure 5D, compare conditions 7 and 8 ).

Antagonist Immunomodulation Effects of Trastuzumab and CAFs in the HER2+ ${ }^{+}$Tumor Ecosystem

Next, we analyzed the effects of trastuzumab on cancer-immune cell interactions. To do so, for each cancer cell, we measured all of adding trastuzumab on an established tumor ecosystem (with CAFs). After $16 \mathrm{hr}$ of co-cultures, moderate flow ( $1 \mu \mathrm{L} / \mathrm{min})$ was applied for $1.5 \mathrm{hr}$ with a syringe pump system to replace the original medium (without the drug) with medium containing trastuzumab (Figure 6A). The cancer-immune interaction times (basal median value $=30 \mathrm{~min}$ ) slowly increased upon the addition of the drug, up to a 2.1-fold increase at 16-20 hr after drug injection (Figure 6B). The analysis of the distributions of cancer-immune interaction times before and after injection of the drug showed that trastuzumab increases the frequency of interactions in the range of 50-125 min (Figure 6C). Shorter interactions may include random collisions and HER2-independent cancer-immune contacts. 
A

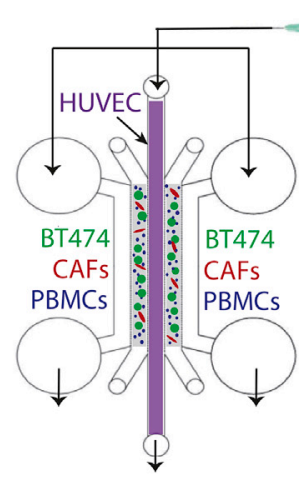

B

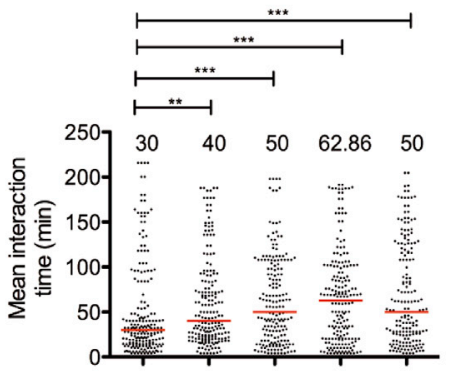

Time interval (hrs) $12-16 \quad 16-20 \quad 24-28 \quad 32-36 \quad 40-44$ $\uparrow$

$t=16$ hrs : start of injection of Trast
Syringe pump-assisted addition of drug

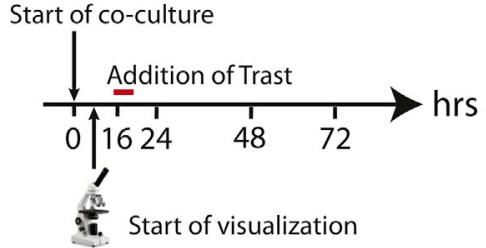

C

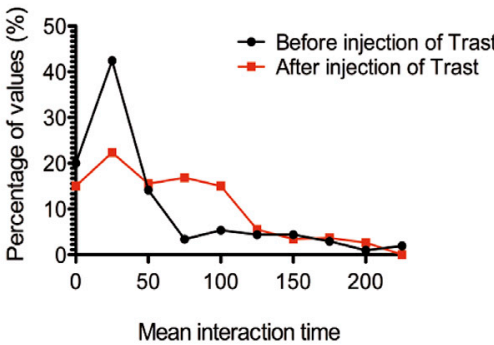

Figure 6. Trastuzumab Promotes Long Cancer-Immune Interactions

(A) Experimental setting. Tumors-on-chip with all 4 cell populations were put under perfusion using a syringe pump. Visualization was started after $12 \mathrm{hr}$ of co-cultures without flow. At $16 \mathrm{hr}, 1 \mu \mathrm{l} / \mathrm{min}$ flow (corresponding to a very moderate $0.03 \mathrm{dyn} / \mathrm{cm}^{2}$ shear stress) was applied for $1.5 \mathrm{hr}$ in the 3 chambers without gels to replace $90 \mu \mathrm{L}$ of the original medium (without drug) with medium containing trastuzumab $(10 \mu \mathrm{g} / \mathrm{mL})$. Flow was then stopped, and visualization was continued up to 3 days.

(B) Kinetics of cancer-immune interactions before and after trastuzumab injection. For each BT474 cancer cell, the mean interaction time (defined as the average of the 100 longest interaction times of the cancer cell with several immune cells) was measured and plotted as 1 point in the graph, using time windows of $4 \mathrm{hr}$. The median values are shown by red bars and explicitly written above the points. Number of cancer cells $n>180$ per time windows. ${ }^{\star} \mathrm{p}<0.05,{ }^{\star \star} \mathrm{p}<0.001,{ }^{\star \star \star} \mathrm{p}<0.0001$; ns, not significant, using Kolmogorov-Smirnov tests. (C) Distribution of cancer-immune interactions before (12-16 hr) and after (24-36 hr) trastuzumab injection.
We re-analyzed the parallelized tumor-on-chip experiments (without flow) with and without drug and with and without CAFs (experimental setting described in Figure 3B), separately considering "short" interactions ( $<50 \mathrm{~min})$ and "long" interactions (>50 min). Figure 7A shows representative results from 1 experiment, which were reproduced in 3 independent experiments, using immune cells from different donors (Figure S5). In the absence of CAFs, the treatment with trastuzumab stimulated the long cancer-immune interactions, but it did not significantly affect the short cancer-immune interactions (Figure 7A, left). Trastuzumab did not stimulate cancer-immune interactions when MCF7 breast cancer cells (not HER2 ${ }^{+}$) were used (Figure $7 \mathrm{~B}$ ), supporting the notion that the observed effects are indeed a consequence of trastuzumab binding the HER2 receptor. The presence of Hs578T CAFs, which we showed as representing the CAF-S1 subtype, inhibited the capacity of trastuzumab to stimulate long cancer-immune cell interactions (Figures $7 \mathrm{~A}$ and S5, right), arguing that CAF-S1 cells, by participating in immunomodulation, may contribute to trastuzumab resistance.

\section{DISCUSSION}

Models have always been of central importance in science since they make a particular part or feature of a complex system easier to understand, define, quantify, visualize, or simulate, by referencing it to existing and commonly accepted knowledge. In this work, we report how to build an experimental model of the tumor ecosystem by using its different "bricks." While the chip platform design is based on previous work (Chen et al., 2017; Lucarini et al.,
2017; Parlato et al., 2017; Zervantonakis et al., 2012), we provide here a significant step forward by simultaneously introducing multiple types of cells (cancer, immune, endothelial, and fibroblasts) and combining the microengineered cancer model with advanced live imaging and automated image analysis, allowing highthroughput tracking at the single-cell level and massive quantifications of cell-cell contacts on-chip. The biological relevance of our tumor microenvironment model is proved by the fact that we did recapitulate ex vivo complex system-level behaviors previously described in in vivo studies, such as the pro-invasive and immunomodulation roles of CAFs, the anti-tumoral ADCC immune response, and the 2 modes of action of trastuzumab (direct action on cancer cells and indirect action via the immune system).

This tumor-on-chip model could be used for basic studies focusing on the tumor microenvironment and for drug preclinical testing within immunocompetent settings. In the era of emerging immunotherapies for cancer (Chen and Mellman, 2017), the technological development of standardized tumors-on-chip could be very valuable to study new oncoimmunotherapies, to increase their efficacy, and to understand the mechanisms of resistance. These tumor-on-chip platforms will be complementary to organotypic tumor spheroids (Jenkins et al., 2018) in that they will provide 2 specific advantages: (1) controllability of each ecosystem parameter (e.g., cell density, ratio between cell types and relative positions, specific cell subsets, local oxygen content, ECM features, vascular perfusion) and (2) direct visualization of cellular dynamics (e.g., proliferation, death, cell-cell interactions, motility) and of their modulations by drugs and ecosystem parameters. 
A
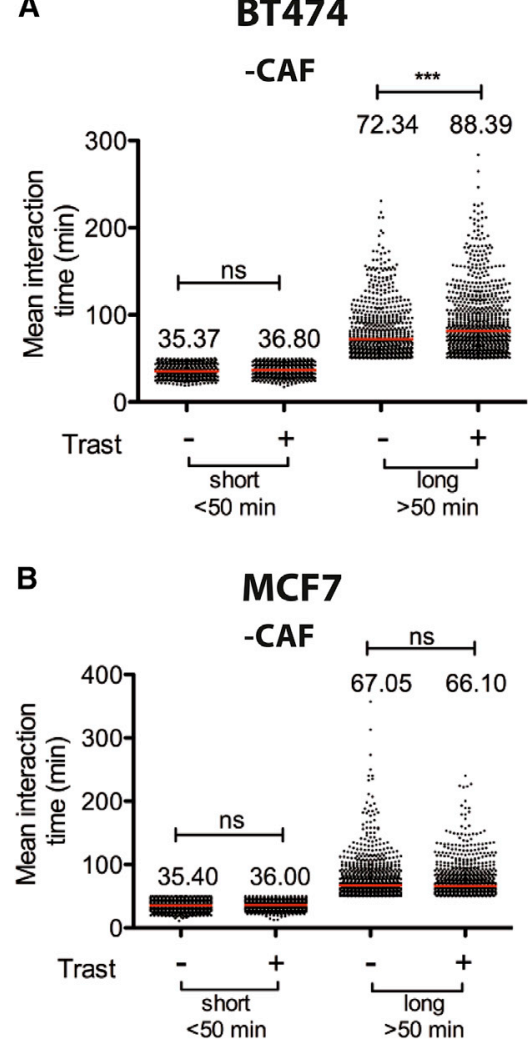

BT474
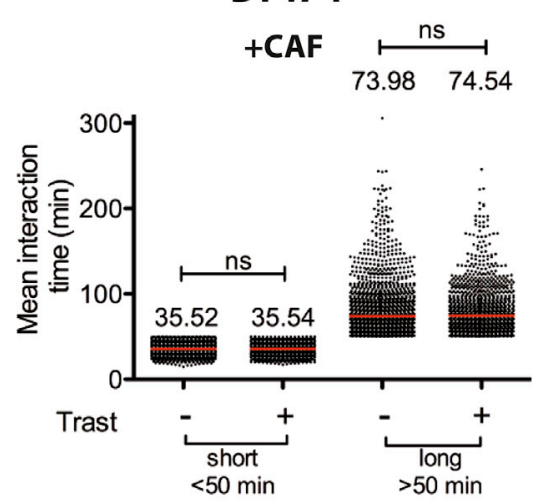

Figure 7. Antagonist Immunomodulation Effects of Trastuzumab and CAFs in the HER2 ${ }^{+}$Tumor Ecosystem

(A) Impact of trastuzumab and CAFs on cancerimmune interactions. The cancer-immune interactions were classified in 2 categories: short (<50 min) and long (>50 min). For each BT474 cancer cell, the mean interaction time was measured and plotted as 1 point in the graph. The median values are shown by red bars and explicitly written above the points. Number of cancer cells $\mathrm{n}>460$ per conditions. ${ }^{*} \mathrm{p}<0.05,{ }^{\star *} \mathrm{p}<0.001,{ }^{* \star *} \mathrm{p}<$ 0.0001 ; ns, not significant, using KolmogorovSmirnov tests.

(B) Trastuzumab does not stimulate the physical interactions between MCF7 cells and immune cells. The tumors-on-chip were generated with MCF7 cells (not HER2 ${ }^{+}$) instead of BT474 cells $\left(\mathrm{HER}^{+}\right)$.

Here, we show that the tumor-on-chip approach turned out to be extremely informative to recapitulate complex features of CAF biology, providing direct visual hints to better understand the crucial interplay of CAFs with both cancer cells (for motility within a matrix) and immune cells (for immunomodulation). The CAF model (Hs578T) used in this work corresponds to the CAF-S1 subtype. In patient samples, CAF-S1 cells were shown to promote an immunosuppressive environment, with accumulation of $\mathrm{FOXP3}^{+} \mathrm{T}$ lymphocytes (Treg) and a concomitant reduction in cytotoxic $\mathrm{CD}^{+} \mathrm{T}$ lymphocytes in breast and ovarian cancers (Costa et al., 2018; Givel et al., 2018). We provide a direct ex vivo visualization of the capacity of immunosuppressive CAF-S1 cells to downmodulate the interactions of cancer cells with PBMCs, which are mainly composed of lymphocytes ( $T$ cells and B cells), natural killer (NK) cells, and monocytes. Even though in this study we could not define the exact identity of the immune cells contacting the cancer cells, since the morphology features are not sufficient to discriminate among the immune cell types, we anticipate that immunocompetent tumors-on-chip will be valuable tools to address questions about the immunosuppressive mechanisms of CAFs.

In addition, our findings provide direct visual evidence for the immune-dependent mechanism of action of trastuzumab: by binding to the overexpressed HER2 receptors on the cancer cell surface, the antibody creates multiple binding sites for Fc receptors that are present on the surface of several immune cell subtypes, including NK cells, B lymphocytes, dendritic cells, and macrophages (Hudis, 2007). This trastuzumab-dependent mated that roughly half of the PBMCs

stimulation in cancer-immune interactions correlated with a stimulation of cancer apoptosis, via ADCC, provided that immune cells were at high density.

A limitation of this $\mathrm{HER}^{+}$tumor-onchip is the short-term viability of PBMC immune cells. By counting the immune cells that underwent apoptosis during the time course of experiments, we estivation time window of a few days was sufficient to visualize and discover some specific features associated with the presence of trastuzumab, immune cells, and CAFs.

Another limitation is the use of allogeneic immune cells that can in principle trigger a major histocompatibility complex (MHC) class I-dependent immune response. However, the incubation time of our co-cultures with immune cells was presumably too short to allow allogeneic reactions to take place; for example, a MLR typically starts after 3 days and is usually measured at 5 days of co-cultures (Nguyen et al., 2003). We consider that the reproducibility of the results with immune cells originating from different donors is a good control. Exploiting the tools and methods developed for this work, it will be possible in the near future to move toward autologous cell models, including primary cells from patients. Since the volume of the microchambers is just a few microliters, tumors-on-chips require an extraordinarily low amount of live cells (in the range of a few thousand), which makes the use of fresh patient-derived samples feasible.

\section{STAR $\star$ METHODS}

Detailed methods are provided in the online version of this paper and include the following:

- KEY RESOURCES TABLE

- CONTACT FOR REAGENT AND RESOURCE SHARING 
- EXPERIMENTAL MODEL AND SUBJECT DETAILS ○ Cell culture

- METHOD DETAILS

O Microfluidic devices

Preparation of tumors-on-chip

o Live cell staining

O Live microscopy

$\bigcirc$ Image analysis

O Flow Cytometry

- QUANTIFICATION AND STATISTICAL ANALYSIS

\section{SUPPLEMENTAL INFORMATION}

Supplemental Information includes one table, five figures, and six videos and can be found with this article online at https://doi.org/10.1016/j.celrep.2018. 12.015.

\section{ACKNOWLEDGMENTS}

We thank Elian Martin for help with the syringe pump and the Cell and Tissue Imaging (PICT-IBiSA), Institut Curie, a member of the French National Research Infrastructure France-Biolmaging (ANR10-INBS-04). This work was supported by Cancéropôle lle-de-France (2015-1-EMERG-10-ICR-1 to M.C.P. and V.S.; no. 2012-2-EML-04-IC-1), Ligue National Contre le Cancer-Comité de Paris (RS14/75-54 to M.C.P.), Fondation pour la Recherche Médicale (ING20160435436 to M.C.P.; DGE20111123020), Institut National de la Santé et de la Recherche Médicale (IINSERM] ITMO Plan Cancer 2014-2018, EQ2016-01, to M.C.P.), INCA (Cancer National Institute, no. 2011-1-LABEL-IC-4), and Association Christelle Bouillot (to J.C.). A.D.N. was supported by the Scientific Independence of Young Researchers Program (SIR 2014) under grant no. RBSI14TX20-MUSIC ("Multidimensional Single-Cell Microfluidic Impedance Cytometry").

\section{AUTHOR CONTRIBUTIONS}

Conceptualization, A.G., V.S., F.M.-G., G.Z., J.C., E.M., L.B., and M.C.P.; Formal Analysis, A.M., F.M.-M., and D.D.G.; Funding Acquisition, A.G., V.S., G.Z., J.C., L.B., and M.C.P.; Investigation, M.N., A.D.N., F.M.-M., G.F., S.S.E., M.C., Y.K., W.H., F.P., and F.R.B.; Methodology, A.D.N., A.M., A.Y., S.D., E.M., and L.B.; Supervision, S.D., V.S., F.M.-G., G.Z., J.C., E.M., L.B., and M.C.P.; Project Administration, M.C.P.; Resources, P.S.; Visualization, M.N., F.M.-M., W.H., and L.B.; Writing - Original Draft, M.C.P.; Writing - Review \& Editing, M.N., A.D.N., A.M., F.M.-M., G.F., S.S.E., M.C., Y.K., W.H., P.S., F.P., D.D.G., F.R.B., A.G., A.Y., S.D., V.S., F.M.-G., G.Z., J.C., E.M., L.B., and M.C.P.

\section{DECLARATION OF INTERESTS}

The authors declare no competing interests.

Received: March 15, 2018

Revised: August 6, 2018

Accepted: December 3, 2018

Published: December 26, 2018

\section{REFERENCES}

Arnould, L., Gelly, M., Penault-Llorca, F., Benoit, L., Bonnetain, F., Migeon, C., Cabaret, V., Fermeaux, V., Bertheau, P., Garnier, J., et al. (2006). Trastuzumab-based treatment of HER2-positive breast cancer: an antibody-dependent cellular cytotoxicity mechanism? Br. J. Cancer 94, 259-267.

Attieh, Y., Clark, A.G., Grass, C., Richon, S., Pocard, M., Mariani, P., Elkhatib, N., Betz, T., Gurchenkov, B., and Vignjevic, D.M. (2017). Cancer-associated fibroblasts lead tumor invasion through integrin- $\beta 3$-dependent fibronectin assembly. J. Cell Biol. 216, 3509-3520.
Bersini, S., Jeon, J.S., Dubini, G., Arrigoni, C., Chung, S., Charest, J.L., Moretti, M., and Kamm, R.D. (2014). A microfluidic 3D in vitro model for specificity of breast cancer metastasis to bone. Biomaterials 35, 2454-2461.

Biselli, E., Agliari, E., Barra, A., Bertani, F.R., Gerardino, A., De Ninno, A., Mencattini, A., Di Giuseppe, D., Mattei, F., Schiavoni, G., et al. (2017). Organs on chip approach: a tool to evaluate cancer -immune cells interactions. Sci. Rep. 7, 12737.

Buchanan, C.F., Szot, C.S., Wilson, T.D., Akman, S., Metheny-Barlow, L.J., Robertson, J.L., Freeman, J.W., and Rylander, M.N. (2012). Cross-talk between endothelial and breast cancer cells regulates reciprocal expression of angiogenic factors in vitro. J. Cell. Biochem. 113, 1142-1151.

Businaro, L., De Ninno, A., Schiavoni, G., Lucarini, V., Ciasca, G., Gerardino, A., Belardelli, F., Gabriele, L., and Mattei, F. (2013). Cross talk between cancer and immune cells: exploring complex dynamics in a microfluidic environment. Lab Chip 13, 229-239.

Chen, D.S., and Mellman, I. (2017). Elements of cancer immunity and the cancer-immune set point. Nature 541, 321-330.

Chen, M.B., Whisler, J.A., Fröse, J., Yu, C., Shin, Y., and Kamm, R.D. (2017). On-chip human microvasculature assay for visualization and quantification of tumor cell extravasation dynamics. Nat. Protoc. 12, 865-880.

Clynes, R.A., Towers, T.L., Presta, L.G., and Ravetch, J.V. (2000). Inhibitory Fc receptors modulate in vivo cytotoxicity against tumor targets. Nat. Med. 6 , 443-446.

Costa, A., Kieffer, Y., Scholer-Dahirel, A., Pelon, F., Bourachot, B., Cardon, M., Sirven, P., Magagna, I., Fuhrmann, L., Bernard, C., et al. (2018). Fibroblast Heterogeneity and Immunosuppressive Environment in Human Breast Cancer. Cancer Cell 33, 463-479.e10.

Davies, E.R. (2004). Machine Vision: Theory, Algorithms, Practicalities (Elsevier).

De Wever, O., Nguyen, Q.-D., Van Hoorde, L., Bracke, M., Bruyneel, E., Gespach, C., and Mareel, M. (2004). Tenascin-C and SF/HGF produced by myofibroblasts in vitro provide convergent pro-invasive signals to human colon cancer cells through RhoA and Rac. FASEB J. 18, 1016-1018.

Diermeier-Daucher, S., Breindl, S., Buchholz, S., Ortmann, O., and Brockhoff, G. (2011). Modular anti-EGFR and anti-Her2 targeting of SK-BR-3 and BT474 breast cancer cell lines in the presence of ErbB receptor-specific growth factors. Cytometry A 79, 684-693.

Feig, C., Jones, J.O., Kraman, M., Wells, R.J.B., Deonarine, A., Chan, D.S., Connell, C.M., Roberts, E.W., Zhao, Q., Caballero, O.L., et al. (2013). Targeting CXCL12 from FAP-expressing carcinoma-associated fibroblasts synergizes with anti-PD-L1 immunotherapy in pancreatic cancer. Proc. Natl. Acad. Sci. USA 110, 20212-20217.

Gaggioli, C., Hooper, S., Hidalgo-Carcedo, C., Grosse, R., Marshall, J.F., Harrington, K., and Sahai, E. (2007). Fibroblast-led collective invasion of carcinoma cells with differing roles for RhoGTPases in leading and following cells. Nat. Cell Biol. 9, 1392-1400.

Givel, A.-M., Kieffer, Y., Scholer-Dahirel, A., Sirven, P., Cardon, M., Pelon, F. Magagna, I., Gentric, G., Costa, A., Bonneau, C., et al. (2018). miR200-regulated CXCL12 $\beta$ promotes fibroblast heterogeneity and immunosuppression in ovarian cancers. Nat. Commun. 9, 1056.

Glentis, A., Oertle, P., Mariani, P., Chikina, A., El Marjou, F., Attieh, Y. Zaccarini, F., Lae, M., Loew, D., Dingli, F., et al. (2017). Cancer-associated fibroblasts induce metalloprotease-independent cancer cell invasion of the basement membrane. Nat. Commun. 8, 924.

Goetz, J.G., Minguet, S., Navarro-Lérida, I., Lazcano, J.J., Samaniego, R., Calvo, E., Tello, M., Osteso-lbáñez, T., Pellinen, T., Echarri, A., et al. (2011). Biomechanical remodeling of the microenvironment by stromal caveolin-1 favors tumor invasion and metastasis. Cell 146, 148-163.

Hassell, B.A., Goyal, G., Lee, E., Sontheimer-Phelps, A., Levy, O., Chen, C.S., and Ingber, D.E. (2017). Human Organ Chip Models Recapitulate Orthotopic Lung Cancer Growth, Therapeutic Responses, and Tumor Dormancy In Vitro. Cell Rep. 21, 508-516.

Heuser, L., Spratt, J.S., and Polk, H.C., Jr. (1979). Growth rates of primary breast cancers. Cancer 43, 1888-1894. 
Hudis, C.A. (2007). Trastuzumab-mechanism of action and use in clinical practice. N. Engl. J. Med. 357, 39-51.

Huh, D., Kim, H.J., Fraser, J.P., Shea, D.E., Khan, M., Bahinski, A., Hamilton, G.A., and Ingber, D.E. (2013). Microfabrication of human organs-on-chips. Nat. Protoc. 8, 2135-2157.

Jamieson, D., Cresti, N., Verrill, M.W., and Boddy, A.V. (2009). Development and validation of cell-based ELISA for the quantification of trastuzumab in human plasma. J. Immunol. Methods 345, 106-111.

Jenkins, R.W., Aref, A.R., Lizotte, P.H., Ivanova, E., Stinson, S., Zhou, C.W., Bowden, M., Deng, J., Liu, H., Miao, D., et al. (2018). Ex Vivo Profiling of PD1 Blockade Using Organotypic Tumor Spheroids. Cancer Discov. 8, 196-215.

Klemm, F., and Joyce, J.A. (2015). Microenvironmental regulation of therapeutic response in cancer. Trends Cell Biol. 25, 198-213.

Kraman, M., Bambrough, P.J., Arnold, J.N., Roberts, E.W., Magiera, L., Jones, J.O., Gopinathan, A., Tuveson, D.A., and Fearon, D.T. (2010). Suppression of antitumor immunity by stromal cells expressing fibroblast activation proteinalpha. Science 330, 827-830.

Kute, T., Stehle, J.R., Jr., Ornelles, D., Walker, N., Delbono, O., and Vaughn, J.P. (2012). Understanding key assay parameters that affect measurements of trastuzumab-mediated ADCC against Her2 positive breast cancer cells. Oncolmmunology 1, 810-821.

Labernadie, A., Kato, T., Brugués, A., Serra-Picamal, X., Derzsi, S., Arwert, E., Weston, A., González-Tarragó, V., Elosegui-Artola, A., Albertazzi, L., et al. (2017). A mechanically active heterotypic E-cadherin/ $\mathrm{N}$-cadherin adhesion enables fibroblasts to drive cancer cell invasion. Nat. Cell Biol. 19, 224-237.

Lee, Y.-C., Gajdosik, M.S., Josic, D., Clifton, J.G., Logothetis, C., Yu-Lee, L.-Y., Gallick, G.E., Maity, S.N., and Lin, S.-H. (2015). Secretome analysis of an osteogenic prostate tumor identifies complex signaling networks mediating cross-talk of cancer and stromal cells within the tumor microenvironment. Mol. Cell. Proteomics 14, 471-483.

Liao, D., Luo, Y., Markowitz, D., Xiang, R., and Reisfeld, R.A. (2009). Cancer associated fibroblasts promote tumor growth and metastasis by modulating the tumor immune microenvironment in a 4T1 murine breast cancer model. PLoS One 4, e7965.

Lucarini, V., Buccione, C., Ziccheddu, G., Peschiaroli, F., Sestili, P., Puglisi, R., Mattia, G., Zanetti, C., Parolini, I., Bracci, L., et al. (2017). Combining Type I Interferons and 5-Aza-2'-Deoxycitidine to Improve Anti-Tumor Response against Melanoma. J. Invest. Dermatol. 137, 159-169.

Marty, M., Cognetti, F., Maraninchi, D., Snyder, R., Mauriac, L., Tubiana-Hulin, M., Chan, S., Grimes, D., Antón, A., Lluch, A., et al. (2005). Randomized phase II trial of the efficacy and safety of trastuzumab combined with docetaxel in patients with human epidermal growth factor receptor 2-positive metastatic breast cancer administered as first-line treatment: the M77001 study group. J. Clin. Oncol. 23, 4265-4274.
Nahta, R., Hung, M.-C., and Esteva, F.J. (2004). The HER-2-targeting antibodies trastuzumab and pertuzumab synergistically inhibit the survival of breast cancer cells. Cancer Res. 64, 2343-2346.

Nguyen, X.D., Eichler, H., Dugrillon, A., Piechaczek, C., Braun, M., and Klüter, H. (2003). Flow cytometric analysis of T cell proliferation in a mixed lymphocyte reaction with dendritic cells. J. Immunol. Methods 275, 57-68.

Parlato, S., De Ninno, A., Molfetta, R., Toschi, E., Salerno, D., Mencattini, A., Romagnoli, G., Fragale, A., Roccazzello, L., Buoncervello, M., et al. (2017). 3D microfluidic model for evaluating immunotherapy efficacy by tracking dendritic cell behaviour toward tumor cells. Sci. Rep. 7, 1093.

Shim, J.S., Rao, R., Beebe, K., Neckers, L., Han, I., Nahta, R., and Liu, J.O (2012). Selective inhibition of HER2-positive breast cancer cells by the HIV protease inhibitor nelfinavir. J. Natl. Cancer Inst. 104, 1576-1590.

Sung, K.E., and Beebe, D.J. (2014). Microfluidic 3D models of cancer. Adv. Drug Deliv. Rev. 79-80, 68-78.

Vacchelli, E., Ma, Y., Baracco, E.E., Sistigu, A., Enot, D.P., Pietrocola, F., Yang, H., Adjemian, S., Chaba, K., Semeraro, M., et al. (2015). Chemotherapyinduced antitumor immunity requires formyl peptide receptor 1 . Science 350, 972-978.

van Duinen, V., Trietsch, S.J., Joore, J., Vulto, P., and Hankemeier, T. (2015). Microfluidic 3D cell culture: from tools to tissue models. Curr. Opin. Biotechnol. 35, 118-126.

Varchetta, S., Gibelli, N., Oliviero, B., Nardini, E., Gennari, R., Gatti, G., Silva, L.S., Villani, L., Tagliabue, E., Ménard, S., et al. (2007). Elements related to heterogeneity of antibody-dependent cell cytotoxicity in patients under trastuzumab therapy for primary operable breast cancer overexpressing Her2. Cancer Res. 67, 11991-11999.

Yang, X., Lin, Y., Shi, Y., Li, B., Liu, W., Yin, W., Dang, Y., Chu, Y., Fan, J., and He, R. (2016). FAP Promotes Immunosuppression by Cancer-Associated Fibroblasts in the Tumor Microenvironment via STAT3-CCL2 Signaling. Cancer Res. 76, 4124-4135.

Yoo, T.-K., Min, J.W., Kim, M.K., Lee, E., Kim, J., Lee, H.-B., Kang, Y.J., Kim, Y.-G., Moon, H.-G., Moon, W.K., et al. (2015). In Vivo Tumor Growth Rate Measured by US in Preoperative Period and Long Term Disease Outcome in Breast Cancer Patients. PLoS One 10, e0144144.

Zervantonakis, I.K., Hughes-Alford, S.K., Charest, J.L., Condeelis, J.S., Gertler, F.B., and Kamm, R.D. (2012). Three-dimensional microfluidic model for tumor cell intravasation and endothelial barrier function. Proc. Natl. Acad. Sci. USA 109, 13515-13520.

Zhang, Y., and Ertl, H.C.J. (2016). Depletion of FAP+ cells reduces immunosuppressive cells and improves metabolism and functions CD8+T cells within tumors. Oncotarget 7, 23282-23299. 


\section{STAR $\star M E T H O D S$}

\section{KEY RESOURCES TABLE}

\begin{tabular}{|c|c|c|}
\hline REAGENT or RESOURCE & SOURCE & IDENTIFIER \\
\hline \multicolumn{3}{|l|}{ Antibodies } \\
\hline anti-CD29-Alexa Fluor 700 & BioLegend & Cat\# 303020; RRID:AB_2130078 \\
\hline anti-CD31-PECy7 & BioLegend & Cat\# 303118; RRID:AB_2247932 \\
\hline anti-CD45-APC-Cy7 & BD Biosciences & Cat\# BD-557833; RRID:AB_396891 \\
\hline anti-CD235a-PerCP/Cy5.5 & BioLegend & Cat\# 349109; RRID:AB_2562705 \\
\hline anti-EpCAM-BV605 & BioLegend & Cat\# 324224; RRID:AB_2562518 \\
\hline anti-FAP & R\&D Systems & Cat\# MAB3715; RRID:AB_2102368 \\
\hline anti-PDGFRb-PE & BioLegend & Cat\# 323606; RRID:AB_2268134 \\
\hline anti-SMA-Alexa Fluor 594 & R\&D Systems & Cat\# IC1420T-025 \\
\hline \multicolumn{3}{|l|}{ Biological Samples } \\
\hline PBMC from healthy donors & $\begin{array}{l}\text { "Etablissement Français du Sang", } \\
\text { Paris, Saint-Antoine Crozatier blood bank }\end{array}$ & $\mathrm{N} / \mathrm{A}$ \\
\hline Breast tumor samples & Institut Curie Hospital & N/A \\
\hline \multicolumn{3}{|l|}{ Chemicals, Peptides, and Recombinant Proteins } \\
\hline Trastuzumab (Herceptin) & Roche & CAS number: $180288-69-1$ \\
\hline Fibronectin human & Sigma & Cat\# F0895 \\
\hline Collagen bovine & Advanced Matrix & Cat\# 5005 \\
\hline CellTraceTMYellow & Thermofisher & Cat\# C34567 \\
\hline CellTraceTMViolet & Thermofisher & Cat\# C34557 \\
\hline CellTraceTMCFSE & Thermofisher & Cat\# C34554 \\
\hline CellTraceTMFar Red & Thermofisher & Cat\# C34564 \\
\hline CellEvent Caspase-3/7 Green Detection Reagent & Thermofisher & Cat\# C10423 \\
\hline siR-DNA, Spirochrome & Tebu Bio & Cat\# SC007 \\
\hline \multicolumn{3}{|l|}{ Critical Commercial Assays } \\
\hline Mycoplasma detection kit, VenorGem Classic & BioValley & Cat\# 11-1250 \\
\hline $\begin{array}{l}\text { Cell authentication kit by SRT profiling, GenePrint } \\
10 \text { system }\end{array}$ & Promega & Cat\# B9510 \\
\hline \multicolumn{3}{|l|}{ Experimental Models: Cell Lines } \\
\hline BT474 & ATCC & Cat\# HTB-20 \\
\hline MCF7 & ATCC & Cat\# HTB-22 \\
\hline Hs578T & ATCC & Cat\# HTB-126 \\
\hline HUVEC & Lonza & Cat\# C2517A \\
\hline \multicolumn{3}{|l|}{ Software and Algorithms } \\
\hline MATLAB & Mathworks Inc., USA & http://www.mathworks.com \\
\hline \multirow[t]{2}{*}{ Cell Hunter (MATLAB Environment) } & Biselli et al., 2017 & \multirow[t]{2}{*}{ Provided by martinelli@ing.uniroma2.it } \\
\hline & Parlato et al., 2017 & \\
\hline Graphpad Prism & $\begin{array}{l}\text { GraphPad Software, La Jolla } \\
\text { California USA }\end{array}$ & www.//graphpad.com \\
\hline FlowJo & FlowJo, LCC & https://www.flowjo.com \\
\hline \multicolumn{3}{|l|}{ Other } \\
\hline Hyclone DMEM medium & GE Healthcare & Cat\# SH30081.01 \\
\hline EBM-2 medium & Lonza & Cat\# CC-3156 \\
\hline MEM medium & Sigma Aldrich & Cat\# M0275 \\
\hline Supplements for growth of Endothelial Cells. & Lonza & Cat\# CC-4176 \\
\hline
\end{tabular}




\section{CONTACT FOR REAGENT AND RESOURCE SHARING}

Further information and requests for resources and reagents should be directed to and will be fulfilled by the Lead Contact, M.C. Parrini (maria-carla.parrini@curie.fr).

\section{EXPERIMENTAL MODEL AND SUBJECT DETAILS}

\section{Cell culture}

The HER2 + cell lines from ductal carcinoma (BT474, ATCC, \#HTB-20), Luminal A adenocarcinoma (MCF7, ATCC, \#HTB-22) and the cancer associated fibroblasts (Hs578T, ATCC, \#HTB-126) were cultured in high-glucose DMEM (GE Healthcare, \#SH30081.01) supplemented with $10 \%$ fetal bovine serum (FBS, Biosera), $1 \%$ Penicillin/Streptomycin (GIBCO), 1\% glutamine (GIBCO). The human umbilical vein endothelial cells (HUVEC, Lonza, \#C2517A) were cultured in EBM-2 medium (Lonza, \#CC-3156) supplemented with a cocktail of growth factors (Lonza, \#CC-4176): hEGF, hydrocortisone, GA-1000 (Gentamicin, Amphotericin-B), FBS 2\%, VEGF, hFGFB, R3-IGF-1, Ascorbic acid, Heparin. All cell lines were systematically tested to exclude mycoplasma contamination using a qPCRbased method (VenorGem Classic, BioValley, \#11-1250) and authenticated by SRT profiling (GenePrint 10 system, Promega, \#B9510). PBMCs were isolated from fresh blood in heparinized tubes from healthy donors by density gradient centrifugation using Lymphoprep (Axis-Shield), according to the local ethical guidelines on experimentation with human samples. Trastuzumab (Herceptin ${ }^{\circledR} 150 \mathrm{mg}$ ) was purchased from Roche. Analysis of tumor samples was performed according to the relevant national law on the protection of people taking part in biomedical research. Human experimental procedures were approved by the Institutional Review Board and Ethics committee of the Institut Curie Hospital group (approval February 12th, 2014) and CNIL (Commission Nationale de l'informatique et des Libertés) ( $N^{\circ}$ approval: 1674356 delivered March 30th, 2013).

\section{METHOD DETAILS}

\section{Microfluidic devices}

The PDMS microfluidic devices were fabricated at the CNR-IFN facility using standard soft-lithography methods. Details of design and microfabrication procedures were reported elsewhere (Chen et al., 2017; Lucarini et al., 2017; Parlato et al., 2017; Zervantonakis et al., 2012).

\section{Preparation of tumors-on-chip}

The microfluidic device was first sterilized in UV-oven ( $365 \mathrm{~nm}$ wavelength) for $30 \mathrm{~min}$ and was coated with $10 \mu \mathrm{g} / \mathrm{mL}$ human fibronectin (Sigma, \# F0895) overnight at $37^{\circ} \mathrm{C}, 5 \% \mathrm{CO}$. The microfluidic device was rinsed with PBS and then dried for at least $5 \mathrm{hr}$ at room temperature in a sterile room. Cells were included in hydrogels of bovine collagen I (PureCol, Advanced Matrix, \#5005) at a final concentration of $2.3 \mathrm{mg} / \mathrm{mL}$, in MEM medium (Sigma Aldrich, \#M0275), containing $0.28 \% \mathrm{NaHCO}(\mathrm{pH}=8$ ) to adjust the final pH to 7 . The targeted final density of BT474, MCF7, Hs578T, PBMCs in gels were respectively $1 \times 10^{6} \mathrm{cells} / \mathrm{mL}, 1 \times 10^{6} \mathrm{cells} / \mathrm{mL}, 0.25 \times 10^{6} \mathrm{cells} / \mathrm{mL}$, and $5 \times 10^{6} \mathrm{cells} / \mathrm{mL}$. HUVECs were loaded in the central chamber at a density of $5 \times 10^{6} \mathrm{cell} / \mathrm{s} / \mathrm{mL}$ and reached confluence to form a monolayer approximately $24 \mathrm{hr}$ after seeding. Common medium (EBM-2 with supplements) was loaded in the 2 most external chambers and never changed to avoid dilution effects.

\section{Live cell staining}

When indicated, fibroblasts were stained with CellTraceTMYellow (Thermofisher \#C34567), endothelial cells with CellTraceTMViolet (Thermofisher \#C34557), cancer cells with CellTraceTMCFSE (Thermofisher \#C34554), immune cells with CellTraceTMFar Red (Thermofisher \#C34564). Cells were trypsinized and re-suspended in solution containing $10 \mu \mathrm{M}$ dye in PBS. Cells were then incubated $30 \mathrm{~min}$ at $37^{\circ} \mathrm{C}, 5 \% \mathrm{CO}$, washed in PBS and loaded in the tumors-on-chip. Cell-event (CellEvent Caspase-3/7 Green Detection Reagent Thermofisher, \#C10423) and siR-DNA (Spirochrome) were added to the chip medium at working concentrations of $6 \mu \mathrm{M}$ and $1 \mu \mathrm{M}$, respectively, to follow live apoptosis and to stain nuclei.

\section{Live microscopy}

Time-lapse videos were acquired interchangeably with two inverted motorized Leica video-microscopes, both equipped with motorized stage, $37^{\circ} \mathrm{C}$ incubator and $\mathrm{CO}_{2}$ controller, using $10 x$ objectives. The first microscope was a Leica DMIRE2 with a Coolsnap HQ2 camera and illumination by mercury Osram lamp at $103 \mathrm{~W}$. The second microscope was a Leica DMi8 with a Retiga R6 camera and illumination by Lumencor SOLA SE 365. The $\mathrm{H}_{2} \mathrm{O}$ saturated atmosphere was obtained by adding wet (sterilized) sponges in close contact with the chips in the incubator. We also added PDMS covers on top of the chips in order to avoid evaporation. The automated imaging system was controlled by the software Metamorph (Universal Imaging). For fluorescence acquisitions, images were taken every $2 \mathrm{hr}$. Transmission images were taken every $2 \mathrm{hr}, 5 \mathrm{~min}$ or $2 \mathrm{~min}$. The number of positions taken per chip varied from 4 to 12 . Day 0 corresponds to the day of the beginning of the co-culture. We started observation under the microscope after 3-24 hr, for 3 days. For confocal imaging we used a Leica SP8 confocal microscope with a 25X water immersion lens (NA: 0.95, WD: 2.4mm); the total scanning depth was $200 \mu \mathrm{m}$ with $3 \mu \mathrm{m}$ in z-step. The captured images were reconstructed in 3-D using software LAS AF. 
Image analysis

The mitotic and apoptotic events were counted by eye on the computer screen, identifying the division of cells in the red channel and the appearance of newly apoptotic cells in the green channel. These events have been normalized by the initial number of cells at day 1. For each experiment and each condition, 3 to 8 positions were counted.

The CellHunter tracking method (Biselli et al., 2017; Parlato et al., 2017) can be divided into two sections: a first block devoted to the differential localization of cells in the image and a second block committed to cell tracking. As pictured in Figure $1 \mathrm{C}$, cell localization is performed by implementing a Circular Hough Transform (CHT) (Davies, 2004) for each frame image in the video. Differential localization is obtained by using different target object radii ( $4 \mu \mathrm{m}$ for PBMCs and $13 \mu \mathrm{m}$ for cancer cells). The second block implements cell tracking. In our application, the clear difference in dimension between immune and cancer cells as well as a not so high cell density in the image allowed for tracking using a Constrained Proximity Tracking (CPT) algorithm. Proximity tracking constructs a track by linking a cell in a frame $i$ with the closest cell in the frame $i+1$, within a maximum distance (radius of the dashed circle pictured in Figure 1C). However, due to the diversified motility characteristics of the two tracked populations of cells, different distances have been considered: $39 \mu \mathrm{m}$ for PBMCs (with high expected motility, this value is about 10 times the radius of the PBMC cell) and $13 \mu \mathrm{m}$ for cancer cells (with moderately lower motility, the value is equal to the tumor cell radius). Of course, when no cells fall within the maximum distance from the given cell (missing tracking) or two different cells are going to be linked to the same cell (conflictual tracking), then the track stops. To solve for unavoidable track splitting phenomena, track gaps procedure has been also considered by allowing missing tracking for at most $10 \mathrm{~min}$. Cell trajectories are then used to conduct a statistical analysis of the physical interactions between PBMCs and cancer cells. We considered two different temporal descriptors, the mean $\left(T_{\text {avg }}\right)$ and the maximum time of interactions $\left(T_{\max }\right)$. These descriptors are estimated from the 'time of interaction' parameter that records the list of all the interactions of a single cancer cell with all the PBMCs using the following procedure: i) For each cancer cell, we identify the starting and the ending frames within which the center of a given PBMC is close to the center of the cancer cell for less than twice the sum of radii of the two cells. ii) The difference between the ending and the starting frames denotes an 'interval of interaction'. By considering all the PBMCs, we collect a list of intervals of interactions for each cancer cell and estimate the so called time of interaction. iii) The maximum interval of interaction, $T_{\max }$, for each cancer cell, represents one of the two statistical descriptors used for the analysis of interaction. iv) To compute the average time of interaction, $T_{\text {avg }}$, first, we sort in decreasing order all the intervals of interaction for each cancer cell; then, we compute the $T_{\text {avg }}$ only considering the first 100 intervals (corresponding to the 100 longest intervals of interaction).

\section{Flow Cytometry}

For surface and intracellular marker staining, cells were analyzed by flow cytometry on the LSRFORTESSA analyzer (BD biosciences), as previously described (Costa et al., 2018). The following antibodies were used to stain the cells in suspension: anti-EpCAM-BV605 (BioLegend, \#324224), anti-CD31-PECy7 (BioLegend, \#303118), anti-CD45-APC-Cy7 (BD Biosciences, \#BD-557833), anti-CD235aPerCP/Cy5.5 (BioLegend, \#349109), anti-CD29-Alexa Fluor 700 (BioLegend, \#303020), anti-FAP (primary antibody, R\&D Systems, \#MAB3715), anti-PDGFRb-PE (BioLegend, \#323606), anti-SMA-Alexa Fluor 594 (R\&D Systems, \#IC1420T-025). We conjugated FAP antibody with fluorescent dye Zenon APC Mouse IgG1 labeling kit (ThermoFisher Scientific, \#Z25051). Data analysis was performed using FlowJo version X 10.0.7r2.

\section{QUANTIFICATION AND STATISTICAL ANALYSIS}

Graphs were created and statistical analysis was performed using Graphpad Prism (v7.0). p values less than 0.05 were considered significant. The statistical details (number of experiments, number of cells or tracks, median or mean) can be found in each figure legend. For cell tracking analysis, the data distributions were assessed by Shapiro-Wilk tests and resulted not normal. Therefore medians were shown and nonparametric Kolmogorov-Smirnov tests were used to evaluate significant differences between groups. 